\title{
Quasi-Orthogonal Space-Time Block Coding Using Polynomial Phase Modulation
}

\author{
Omar Granados and Jean Andrian \\ Department of Electrical and Computer Engineering, Florida International University, 10555 West Flagler Street, Miami, \\ FL 33174, USA \\ Correspondence should be addressed to Jean Andrian, andrianj@fiu.edu
}

Received 8 March 2011; Accepted 28 April 2011

Academic Editors: A. Maaref, S. S. Pietrobon, and A. Vaccaro

Copyright ( 2011 O. Granados and J. Andrian. This is an open access article distributed under the Creative Commons Attribution License, which permits unrestricted use, distribution, and reproduction in any medium, provided the original work is properly cited.

\begin{abstract}
Recently, polynomial phase modulation (PPM) was shown to be a power- and bandwidth-efficient modulation format. These two characteristics are in high demand nowadays specially in mobile applications, where devices with size, weight, and power (SWaP) constraints are common. In this paper, we propose implementing a full-diversity quasi-orthogonal space-time block code (QOSTBC) using polynomial phase signals as modulation format. QOSTBCs along with PPM are used in order to improve the power efficiency of communication systems with four transmit antennas. We obtain the optimal PPM constellations that ensure full diversity and maximize the QOSTBC's minimum coding gain distance. Simulation results show that by using QOSTBCs along with a properly selected PPM constellation, full diversity in flat fading channels and thus low BER at high signal-to-noise ratios (SNR) can be ensured. More importantly, it is also shown that QOSTBCs using PPM achieve a better error performance than those using conventional modulation formats.
\end{abstract}

\section{Introduction}

Orthogonal space-time block codes (STBCs) have drawn a great deal of attention from researchers in recent years. Due to the diversity gain they provide to communication systems, these codes are used to more effectively exploit the multipath characteristics of the wireless channel [1]. It has been shown, however, that orthogonal codes for complex constellations and more than two transmit antennas cannot achieve full transmission rate. To address this issue, Jafarkhani in [2], proposed a code structure in which the columns of the transmission matrix are divided into groups. While the columns within a group of columns are not orthogonal to each other, different groups of columns are orthogonal to each other. This code structure is known as quasi-orthogonal STBCs (QOSTBCs) and although such codes guarantee full transmission rate, they sacrifice full diversity.

In [3] Su and Xia presented quasi-orthogonal STBCs that attain full rate and full diversity. Such characteristics can be readily achieved by optimally selecting a constellation rotation angle subject to the maximization of the metric known as diversity product. Specifically, half of the symbols transmitted in one signaling interval are chosen from the unmodified constellation, while the other half are chosen from the optimally rotated constellation. In [3], the optimal rotation angle for PSK and QAM constellations was presented, and it was demonstrated that the QOSTBCs employing constellation rotation have an improved bit error rate (BER) performance in comparison with the QOSTBCs without it.

The codes presented in [3] make use of optimally rotated PSK and QAM constellations to achieve full diversity; however, for wireless communication devices with size, weight, and power (SWaP) constraints one also has to ensure the most power-efficient modulation format is used. To address this limitation in single-antenna systems, in [4], Sinha et al. introduced a modulation format in which the phase coefficients of polynomial phase functions are used to carry information. This modulation format, known as polynomial phase modulation (PPM), was shown to provide significant improvements in BER performance under additive white Gaussian noise (AWGN) and Rayleigh fading 
channels compared to PSK constellations. The demodulation of PPM signals is carried out using the so-called high-order ambiguity function (HAF) described in $[5,6]$. Following the results in [4] for single input single output (SISO) systems, Dam et al. in [7] proposed a space-time module structure for PPM signals. The proposed module structure allows for the simple design of real orthogonal space-time codes for more than two transmit antennas because the encoding is performed on the phase coefficients. Nevertheless, in [7], it is also shown that the error rate performance of such structure is worse than conventional modulation formats at high signal-to-noise ratios (SNR). The reason behind this decline in performance is that by encoding only the phase coefficients, the module structure does not ensure that the transmitted signals will provide full transmit diversity.

In this paper, in contrast with [7], we design full-diversity PPM systems using QOSTBCs for four transmit antennas by encoding the transmitted signal. In order to guarantee the maximum diversity for the proposed systems, the QOSTBC structure presented by Jafarkhani in [2] is used to encode optimally rotated PPM constellations. Here, we obtain the optimal rotation angle for binary PPM signal constellations. Then, through Monte-Carlo simulations, it is shown that rotating the constellation by the optimal angle yields an improved bit error rate (BER) performance compared with nonrotated PPM constellations. In these simulations, we also show that the proposed systems perform better in terms of error rate than conventional systems using PSK modulation.

This paper is organized as follows. In Section 2, the system and polynomial phase modulation (PPM) signal model are presented. In Section 3, the Jafarkhani scheme for quasi-orthogonal space-time codes is described as well as its condition for full diversity. In Section 4, the optimal constellations for the proposed modulation format using different polynomial phase orders are obtained. Simulation of the error rate performance of a QOSTBC using different PPM constellations is presented in Section 5. Concluding remarks are presented in Section 6.

\section{System and Signal Model}

For a system with $N$ transmit and $M$ receive antennas, the received signal $r_{k, m}$ at time instant $k$ and antenna $m$ is given by

$$
r_{k, m}=\sum_{n=1}^{N} h_{n, m} C_{k, n}+\eta_{k, m}
$$

where $1 \leq k \leq T$ and each coefficient of the $T \times N$ transmission matrix $C$ is denoted as $C_{k, n}$. The coefficients $h_{n, m}$ are independent identically distributed (i.i.d) complex Gaussian random variables which represent the components of an $N \times$ $M$ quasistatic flat fading wireless channel. Finally, $\eta_{k, m}$ are the components of a $T \times M$ matrix of independent samples of a zero mean complex Gaussian random variable.

In this paper, a wireless communication system with four transmit antennas and one receive antenna is considered for simplicity; however, the system can be easily extended to more than one receive antenna. The modulation format used here is known as polynomial phase modulation and the modulated signal $s(t)$ for a symbol period $T_{0}$ is

$$
s(t)=A \exp \left(j\left(\omega_{c} t+\varphi(t)\right)\right)
$$

where $A$ is the signal amplitude and is assumed to be unity, $0 \leq t \leq T_{0}$, and

$$
\varphi(t)=a_{L}\left(\frac{t}{T_{0}}\right)^{L}+a_{L-1}\left(\frac{t}{T_{0}}\right)^{L-1}+\cdots+a_{1}\left(\frac{t}{T_{0}}\right)+a_{0}
$$

is the time-varying phase whose phase coefficients are chosen as $a_{i} \in\{ \pm \pi / 2\}$. Therefore, depending on the transmitted symbol, a specific combination of phase coefficients is selected. For instance, given a linear-binary PPM signal, if the symbol to be transmitted is 01 , the selected phase coefficients are $a_{(1)}=-\pi / 2, a_{0}=\pi / 2$. It is important to note that if a higher transmission rate is required, higher polynomial phase orders could be employed. The system block diagram is illustrated in Figure 1. In the diagram, it is shown that the input binary stream is mapped onto a sequence of PPM symbols. Each set of four symbols $\left(s_{1}, s_{2}, s_{3}, s_{4}\right)$ is then transformed by the $T \times N$ transmission matrix $C$. At the receiver end, the signal is decoded through pairwise maximum likelihood (ML) decoding to recover the original bit stream.

\section{STBC from Quasi-Orthogonal Design}

Since full transmission rate cannot be accomplished with complex orthogonal STBCs when more than two transmit antennas are used, the quasi-orthogonal STBC proposed by Jafarkhani in [2] is employed.

For two transmit antennas, Alamouti [1] proposed the following full rate orthogonal STBC for complex signal constellations,

$$
G\left(s_{1}, s_{2}\right)=\left(\begin{array}{cc}
s_{1} & s_{2} \\
-s_{2}^{*} & s_{1}^{*}
\end{array}\right)
$$

Then, for four transmit antennas Jafarkhani expanded the Alamouti scheme to

$$
C=\left(\begin{array}{cc}
G\left(s_{1}, s_{2}\right) & G\left(s_{3}, s_{4}\right) \\
-G^{*}\left(s_{3}, s_{4}\right) & G^{*}\left(s_{1}, s_{2}\right)
\end{array}\right) .
$$

From (4) and (5), it follows that

$$
C=\left(\begin{array}{cccc}
s_{1} & s_{2} & s_{3} & s_{4} \\
-s_{2}^{*} & s_{1}^{*} & -s_{4}^{*} & s_{3}^{*} \\
-s_{3}^{*} & -s_{4}^{*} & s_{1}^{*} & s_{2}^{*} \\
s_{4} & -s_{3} & -s_{2} & s_{1}
\end{array}\right) .
$$

The block code shown in (6) clearly achieves full rate as one symbol is transmitted per time interval. Nevertheless, due to the relaxation of the code orthogonality requirement, decoding complexity increases with respect to that of 


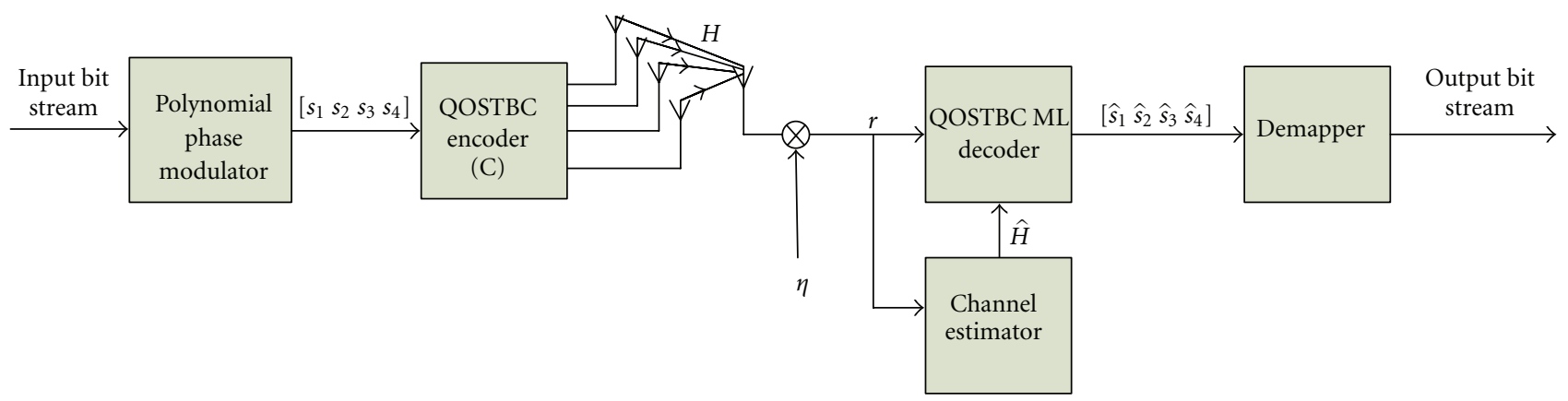

Figure 1: System block diagram.

orthogonal codes because only pairwise decoding is possible. Specifically, decoding QOSTBC encoded signals is reduced to minimizing the cost functions over all possible pairs of symbols [2]:

$$
\begin{aligned}
f_{14}\left(s_{1}, s_{4}\right)= & \left(\left|s_{1}\right|^{2}+\left|s_{4}\right|^{2}\right)\left(\sum_{n=1}^{4}\left|h_{n}\right|^{2}\right) \\
& +2 \Re\left\{\left(-h_{1} r_{1}^{*}-h_{2}^{*} r_{2}-h_{3}^{*} r_{3}-h_{4} r_{4}^{*}\right) s_{1}\right. \\
& \left.+\left(-h_{4} r_{1}^{*}+h_{3}^{*} r_{2}+h_{2}^{*} r_{3}-h_{1} r_{4}^{*}\right) s_{4}\right\} \\
& +4 \Re\left\{h_{1} h_{4}^{*}-h_{2}^{*} h_{3}\right\} \Re\left\{s_{1} s_{4}^{*}\right\}, \\
f_{23}\left(s_{2}, s_{3}\right)= & \left(\left|s_{2}\right|^{2}+\left|s_{3}\right|^{2}\right)\left(\sum_{n=1}^{4}\left|h_{n}\right|^{2}\right) \\
& +2 \Re\left\{\left(-h_{2} r_{1}^{*}+h_{1}^{*} r_{2}-h_{4}^{*} r_{3}+h_{3} r_{4}^{*}\right) s_{2}\right. \\
& \left.+\left(-h_{3} r_{1}^{*}-h_{4}^{*} r_{2}+h_{1}^{*} r_{3}+h_{2} r_{4}^{*}\right) s_{3}\right\} \\
& +4 \Re\left\{h_{2} h_{3}^{*}-h_{1}^{*} h_{4}\right\} \Re\left\{s_{2} s_{3}^{*}\right\} .
\end{aligned}
$$

In the proposed QOSTBC PPM system, the binary information is recovered using a maximum likelihood algorithm that estimates the polynomial phase signal parameters of the symbols estimated through (7).

Aside from the decoding complexity, another important limitation of quasi-orthogonal STBCs is their lower BER performance at high SNRs compared to orthogonal STBCs. The reason for this is that QOSTBCs, if generated from only one constellation, do not comply with the full diversity requirement described in the following manner.

Given a pair of distinct transmitted codewords

$$
C=C\left(s_{1}, s_{2}, \widetilde{s_{3}}, \widetilde{s_{4}}\right), \quad C^{\prime}=C\left(s_{1}^{\prime}, \tilde{s}_{2}^{\prime}, \tilde{s_{3}^{\prime}}, \tilde{s_{4}^{\prime}}\right),
$$

where $\tilde{s_{3}}$ and $\tilde{s_{4}}$ are rotated versions of $s_{3}$ and $s_{4}$, the coding gain distance (CGD) is defined as

$$
\mathrm{CGD}=\operatorname{det}\left[D\left(C, C^{\prime}\right)^{H} D\left(C, C^{\prime}\right)\right],
$$

where $D\left(C, C^{\prime}\right)$ is $\left(C^{\prime}-C\right)$ and $D(\cdot)^{H}$ is the Hermitian of $D(\cdot)$. From this, full diversity can be achieved if and only if the above determinant is not zero. For this condition to uphold, one should choose symbols $s_{1}$ and $s_{2}$ from the regular constellation and $\widetilde{s_{3}}$ and $\widetilde{s_{4}}$ from a different one. The second constellation, in this case, should be chosen such that the minimum CGD is maximized. For a signal constellation $\mathcal{A}$, this optimization problem is described in [8] as

$$
\operatorname{CGD}_{\min }(\phi(t))=\max _{\left(s_{1}, \tilde{s}_{3}\right) \neq\left(s_{1}^{\prime}, \tilde{s}_{3}^{\prime}\right)}\left|\left(s_{1}-s_{1}^{\prime}\right)^{2}-\left(\tilde{s_{3}}-\tilde{s_{3}^{\prime}}\right)^{2}\right|^{4},
$$

where $s_{1} \in \mathcal{A}$ and $\tilde{s_{3}} \in e^{j \varnothing(t)} \mathcal{A}$.

If $d_{\min }=\min \left(\left|s_{1}-s_{1}^{\prime}\right|\right)$, then the upper bound of the minimum CGD can be expressed as [8]

$$
\mathrm{CGD}_{\min }(\phi(t)) \leq d_{\min }^{8} .
$$

\section{Optimal PPM Constellations}

As mentioned in the previous section, full diversity can be achieved if symbols $\widetilde{s_{3}}$ and $\widetilde{s_{4}}$ are chosen from a constellation rotated with respect to the one used for symbols $s_{1}$ and $s_{2}$. The optimization problem is thus to select the proper rotation angle function, $\varnothing(t)$, that maximizes the minimum CGD defined in (10).

Then, for the case of linear binary polynomial phase modulation, where the phase coefficients are chosen from $a_{i} \in\{ \pm \pi / 2\}$, the rotation angle function considered is of the form

$$
\varnothing(t)=\varnothing_{1}\left(\frac{t}{T_{0}}\right)+\varnothing_{0},
$$

where $\varnothing_{1}$ and $\varnothing_{0}$ are the rotation angles for the firstorder and constant phase coefficients, respectively, and can be chosen from the range $0 \leq \varnothing_{i} \leq \pi / 2$. Since the PPM signals are dependent on time, it is important to notice that for the symbol decoding process in (7) and the optimization problem in (10), the distance, $d$, between a pair of distinct continuous-time PPM signals is calculated using

$$
d^{2}=\int_{0}^{T_{0}}\left(s_{m}(t)-s_{n}(t)\right)^{2} d t .
$$

Based on this, the surface plot of the minimum CGD for linear-binary PPM using different rotation angles was 


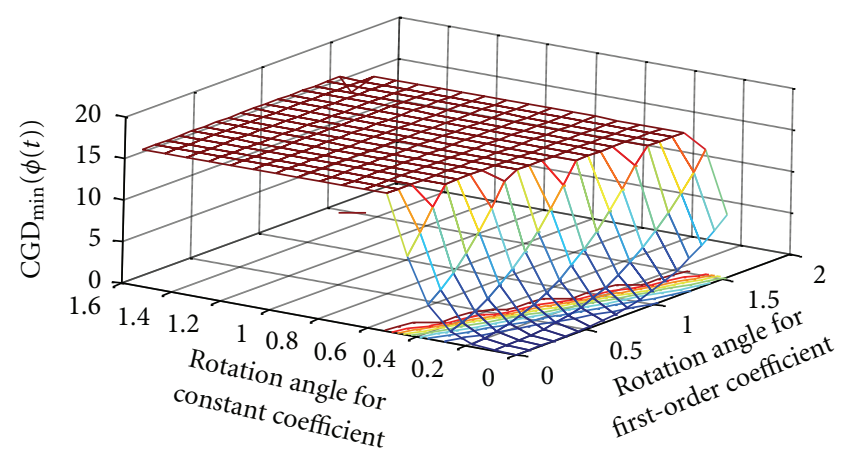

Figure 2: $\mathrm{CGD}_{\min }(\phi(t))$ for rotations on the constant and firstorder coefficients.

TABLE 1: Comparison of CGD $\min$ values for PPM and PSK constellations.

\begin{tabular}{lcccc}
\hline Constellation & $\begin{array}{c}\text { Linear-binary } \\
\text { PPM }\end{array}$ & QPSK & $\begin{array}{c}\text { Quadratic- } \\
\text { binary } \\
\text { PPM }\end{array}$ & 8-PSK \\
\hline $\mathrm{CGD}_{\min }$ & 16 & 16 & 0.0100 & 0.0404 \\
$d_{\min }^{8}$ & 16 & 16 & 0.0100 & 0.1178 \\
\hline
\end{tabular}

generated, and it is shown in Figure 2. In this figure, it can be seen that the rotation angle function that maximizes the minimum CGD is not unique; however, one can observe that a valid and convenient choice would be to pick $\varnothing_{1}=0$ and $\varnothing_{0}=\pi / 2$. As a result, even though the information bits are being carried on both the linear and constant phase coefficients, the highest value of $\mathrm{CGD}_{\min }$ can be attained by employing a constellation in which only the constant phase coefficient is rotated.

Let us now consider the case in which the constellation consists of binary quadratic PPM signals where, again, the phase coefficients are selected from $a_{i} \in\{ \pm \pi / 2\}$. For this particular instance, we optimize (8) based on the following quadratic rotation angle function:

$$
\varnothing(t)=\varnothing_{2}\left(\frac{t}{T_{0}}\right)^{2}+\varnothing_{1}\left(\frac{t}{T_{0}}\right)+\varnothing_{0}
$$

Using the above function, the optimization problem in (10) was solved numerically. It was found that by setting $\varnothing_{2}=0, \varnothing_{1}=0$, and $\varnothing_{0}=\pi / 2$ the upper bound of the $\mathrm{CGD}_{\min }$ was obtained. In consequence, only the constant phase coefficient of the optimal constellation needs to be rotated in order to maximize the coding gain distance. This result is the same as the one obtained for binary linear PPM, and, as shown in Table 1, both constellations achieve the $\mathrm{CGD}_{\min }$ upper bound described in (11). From this, we can infer that an optimal rotation angle for binary PPM constellations of any order is $\pi / 2$. As a way of validating these results, in the following section, we evaluate the error rate for linear and quadratic binary PPM using Monte Carlo simulations.

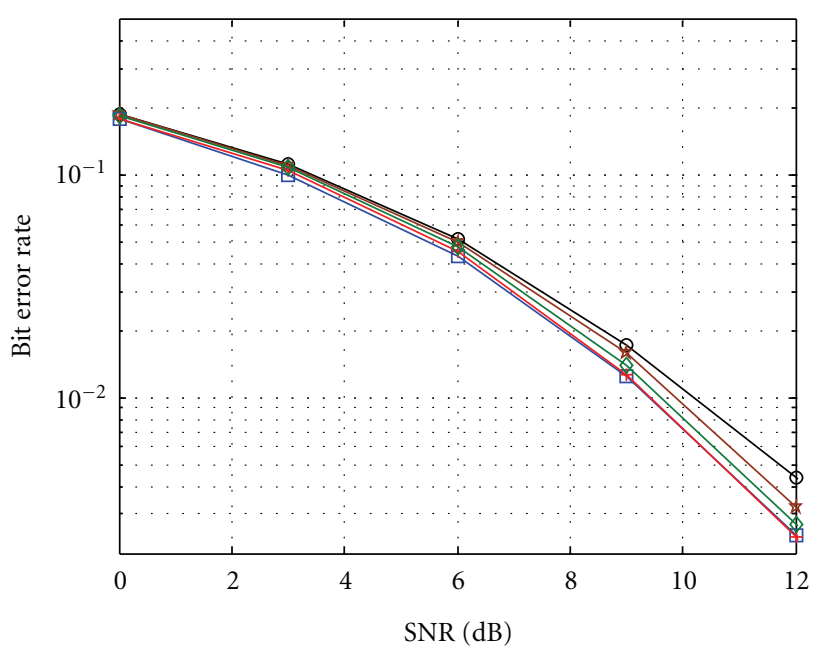

$\multimap$ Linear-binary PPM $(\phi=0) \quad \longrightarrow$ Linear-binary PPM $(\phi=1)$

$\rightarrow$ Linear-binary $\operatorname{PPM}(\phi=\pi / 10) \smile$ Linear-binary $\operatorname{PPM}(\phi=0.6)$

$\square$ Linear-binary $\operatorname{PPM}(\phi=\pi / 2)$

FIGURE 3: BER for binary linear PPM with QOSTBC for different rotation angles.

\section{Results and Discussion}

In the following Monte Carlo simulations, the BER performance of PPM using the Jafarkhani [2] QOSTBC for systems with four antennas at the transmitter and one antenna at the receiver was studied. For these simulations, we used systems using binary linear and quadratic PPM constellations. We also assumed that the coefficients of the Rayleigh flat fading channel were constant during one block of code transmission and were known at the receiver. In addition to this, we used a ML demodulator to estimate the PPM signal parameters, and the number of samples taken from the signals was 32.

In Figure 3, the error rate for a QOSTBC system with a binary linear PPM constellation using different rotation angles is shown. There, it can be seen that the system with a rotation angle of $\pi / 2$ displays a higher diversity order than for any other rotation angle. Specifically, we can notice that the system using the nonrotated constellation has the highest BER for high values of signal-to-noise ratio (SNR). Next, in Figure 4, the performance of the proposed full diversity QOSTBC using linear-binary PPM is compared with that of a QOSTBC system using QPSK. The error rate in Rayleigh fading channels is lower for linear PPM than for QPSK and, as shown in the figure, the proposed system clearly outperforms the system using QPSK in terms of their error rate performance. We can see that at an error rate of $10^{-4}$, the SNR gap between both modulation schemes is about $1 \mathrm{~dB}$ making the linear-binary PPM system a more power-efficient alternative than the QOSTBC using QPSK.

Afterwards, the BER performance of binary quadratic PPM for different rotation angles was evaluated and presented in Figure 5. As expected from the discussion in the previous section, the optimal rotation, $\pi / 2$, yields the highest diversity order and thus, the lowest error rate. Lastly, we compared the proposed full-diversity scheme with one 


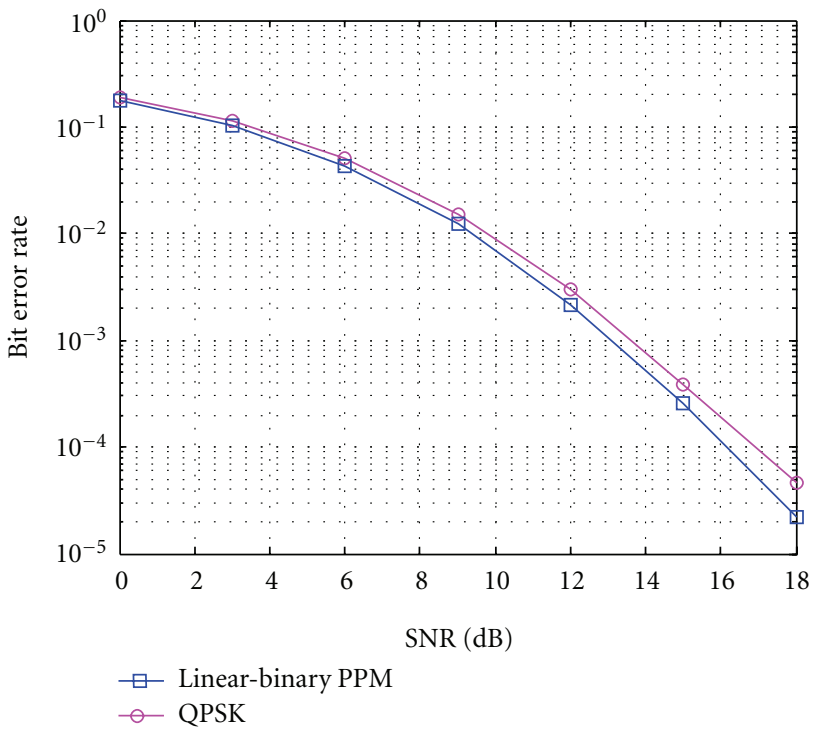

FIgURE 4: BER for QOSTBC using binary linear PPM and QPSK.

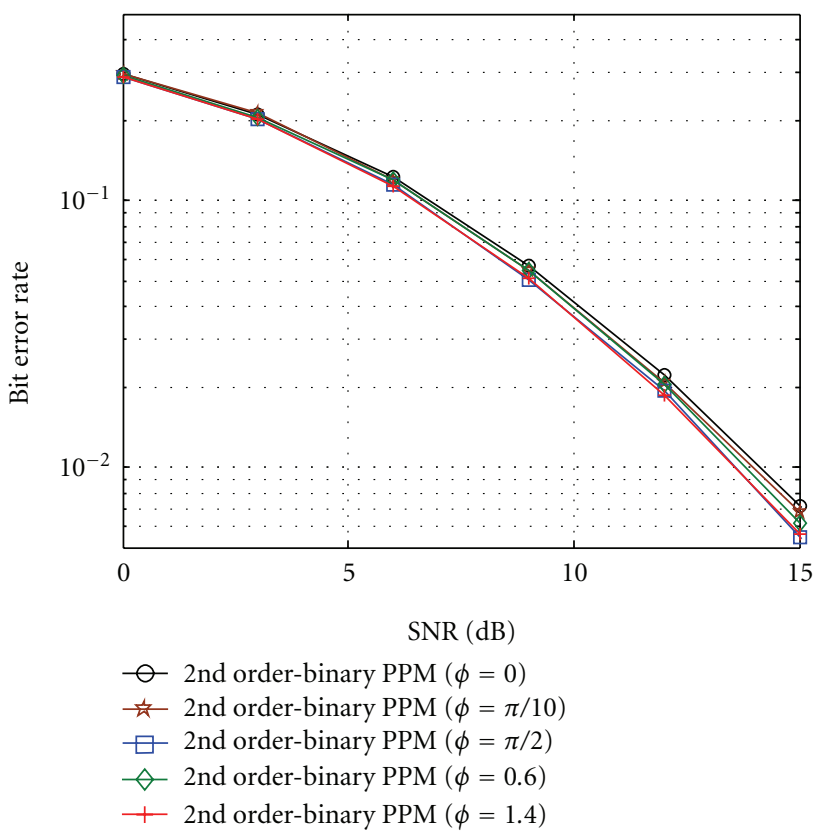

FIGURE 5: BER for QOSTBC using binary quadratic PPM for different rotation angles.

employing optimally rotated 8-PSK constellation. Again, we observe that at an error rate of $10^{-2}$, the system using 8-PSK requires approximately $1.5 \mathrm{~dB}$ more power than the proposed system.

\section{Conclusions}

In this paper, we proposed to make use of PPM-modulated signals and QOSTBCs with the aim of improving the BER performance and, thus, the power efficiency of multiple antenna systems. However, in order to take advantage of the

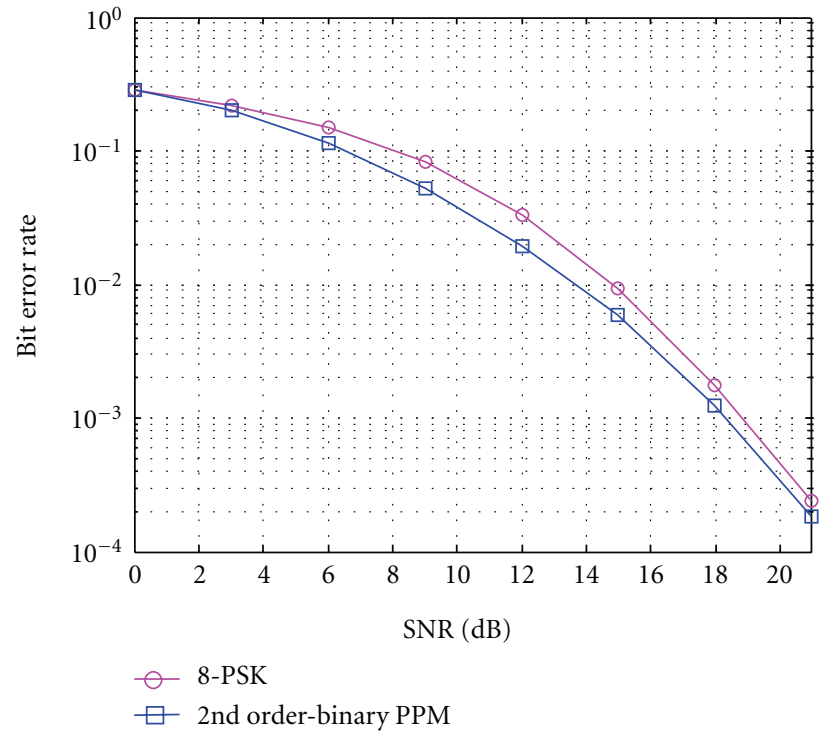

FIGURE 6: BER for QOSTBC using full-diversity 8-PSK and binary quadratic PPM.

multipath characteristics of the channel, full diversity must be ensured. Also, it was noted that the highest diversity order can only be achieved by selecting half of the symbols from an optimally rotated constellation. Therefore, the optimal rotation angles for binary linear and quadratic PPM were obtained by maximizing the system's minimum CGD. Here, we found that binary PPM signals of any order achieve the maximum diversity when the rotation angle is $\pi / 2$. Then, through Monte-Carlo simulations we showed that by using the optimal rotation angle, high-diversity order and thus an improved BER performance at high SNRs can be attained. In addition, it was demonstrated that the proposed fulldiversity PPM-QOSTBC systems outperform systems using conventional PSK constellations.

\section{References}

[1] S. M. Alamouti, "A simple transmit diversity technique for wireless communications," IEEE Journal on Selected Areas in Communications, vol. 16, no. 8, pp. 1451-1458, 1998.

[2] H. Jafarkhani, "A quasi-orthogonal space-time block code," IEEE Transactions on Communications, vol. 49, no. 1, pp. 1-4, 2001.

[3] W. Su and X. G. Xia, "Signal constellations for quasi-orthogonal space-time block codes with full diversity," IEEE Transactions on Information Theory, vol. 50, no. 10, pp. 2331-2347, 2004.

[4] R. Sinha, G. E. Atkin, and C. Zhou, "Performance evaluation of variable-rate polynomial phase modulation," in Proceedings of the Military Communications Conference (MILCOM '07), pp. 1-7, IEEE, Orlando, Fla, USA, October 2007.

[5] S. Barbarossa and A. Scaglione, "Demodulation of CPM signals using piecewise polynomial-phase modeling," in Proceedings of the International Conference on Acoustics, Speech and Signal Processing, vol. 6, pp. 3281-3284, IEEE, May 1998.

[6] Y. Jianming and G. B. Giannakis, "Product multi-lag high-order ambiguity function for blind equalization of polynomial phase signals," in Proceedings of the IEEE-SP International Symposium 
on Time-Frequency and Time-Scale Analysis, pp. 529-532, Paris, France, June 1996.

[7] H. P. Dam, L. Huang, and G. E. Atkin, "Mimo/-space time codes for Polynomial Phase modulation wireless communications," in Proceedings of the Military Communications Conference (MILCOM '09), pp. 1-7, IEEE, Boston, Mass, USA, October 2009.

[8] H. Jafarkhani, Space-Time Coding: Theory and Practice, Cambridge University Press, 2005. 

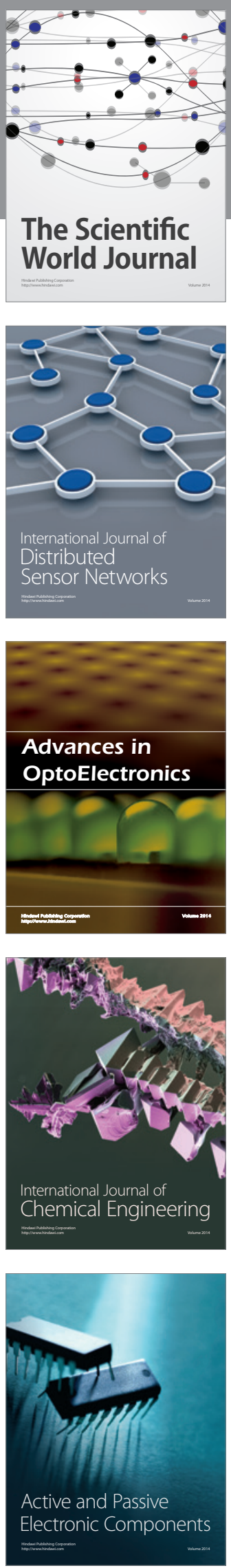
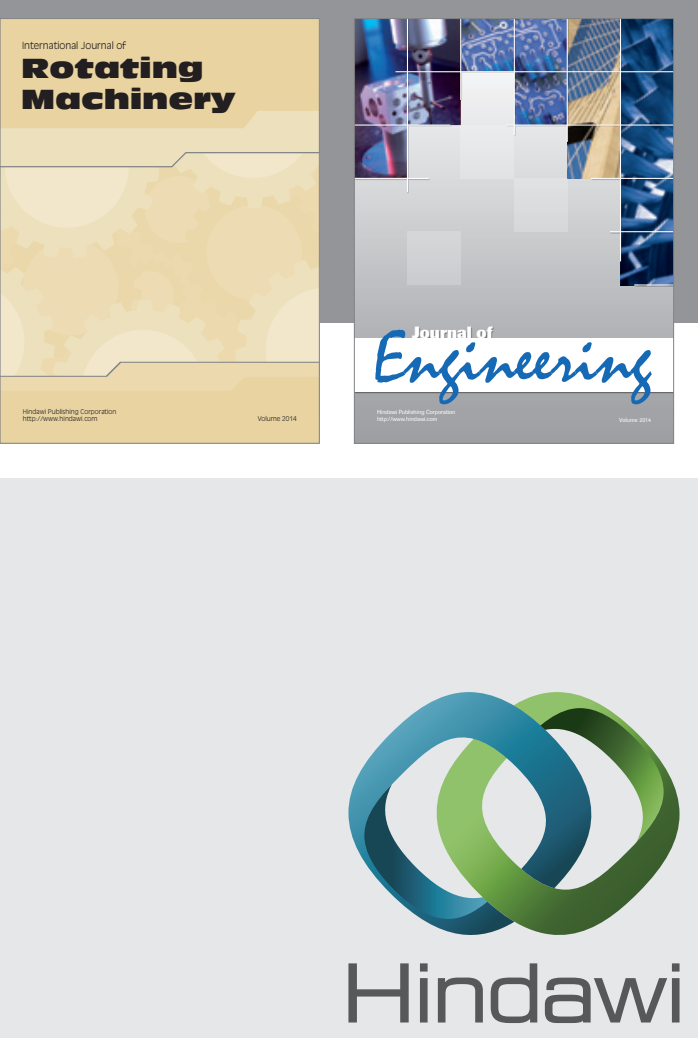

Submit your manuscripts at

http://www.hindawi.com
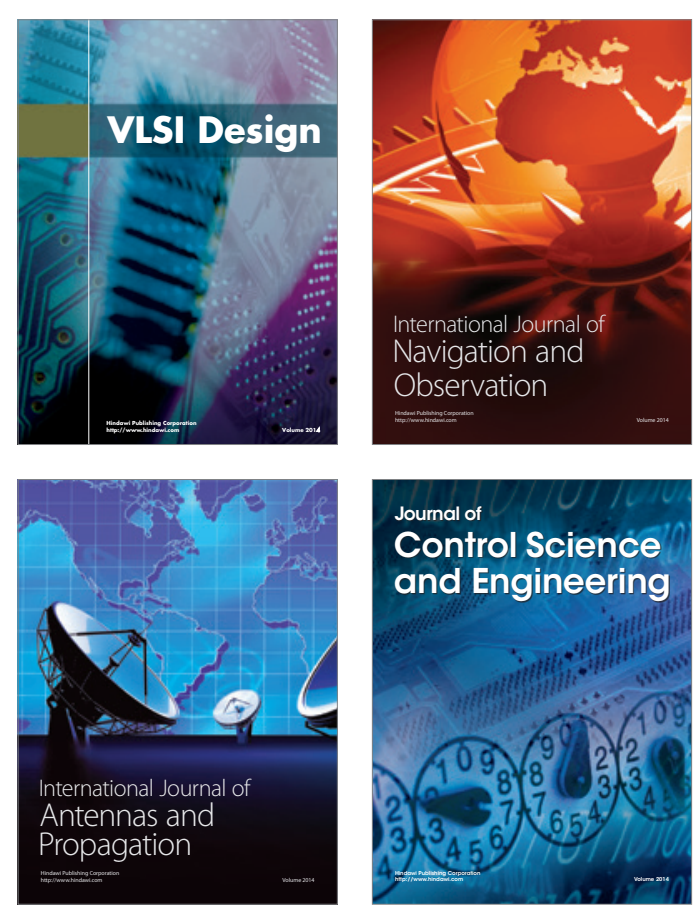
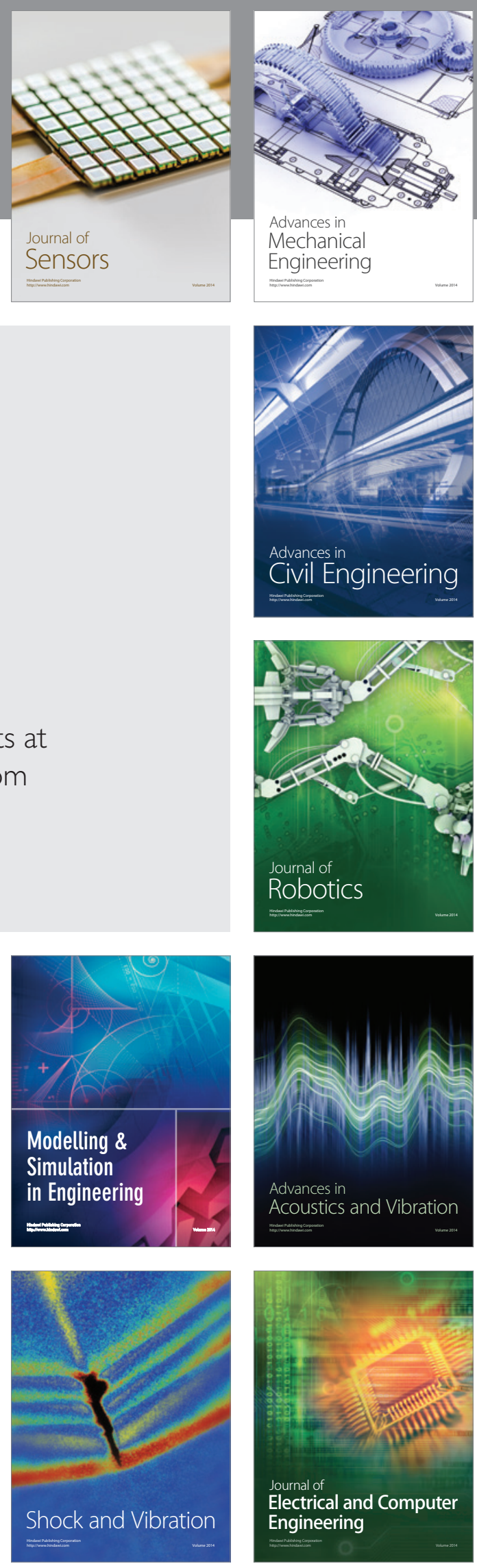